\title{
Decreased visual acuity in patients with cytomegalovirus retinitis and AIDS
}

\begin{abstract}
Purpose To investigate the causes of decreased visual acuity in patients with cytomegalovirus (CMV) retinitis in the acquired immunodeficiency syndrome (AIDS). Methods All human immunodeficiency virus (HIV)-positive patients seen in two ophthalmology units over a 15 month period from September 1996 were included in this retrospective study. A detailed ophthalmic examination was performed on all patients and in addition those with CMV retinitis underwent serial fundus photography.

Decreased visual acuity was defined as a best corrected visual acuity $\leqslant 6 / 12$. CMV and retroviral treatment, $\mathrm{CD} 4^{+}$count and $\mathrm{HIV}$ viral load were also documented for each patient. Results Of 110 patients seen over the 15 month period, 26 (41 eyes) had a diagnosis of CMV retinitis. Twelve patients (16 eyes) with CMV retinitis had decreased visual acuity. The decreased visual acuity in 7 eyes was initially due to the CMV retinitis involving the macula and the optic nerve. Retinal detachment was responsible in 2 eyes and optic nerve atrophy in 1 eye. In 6 eyes (4 patients) the decreased visual acuity was due to a maculopathy - cystoid macular oedema and/or an epiretinal membrane in the presence of an inactive zone 2 or $3 \mathrm{CMV}$ retinitis - with all these patients exhibiting a vitritis of varying grade. The decreased visual acuity in the maculopathy subgroup was irreversible in all except 1 eye, and 2 eyes in this category later developed a cataract.

Conclusion In this series, CMV-retinitis'related' maculopathy was a major $(38 \%)$ cause of decreased visual acuity, occurring in the absence of zone 1 retinitis and despite inactive peripheral CMV retinitis. A varying degree of vitritis was an associated feature in all these patients. This study therefore highlights maculopathy as an important and previously unrecognised significant cause of visual morbidity in CMV retinitis.
\end{abstract}

Key words Acquired immunodeficiency syndrome, Cytomegalovirus retinitis, Macular oedema, Visual acuity
J. RAINA, J.W.B. BAINBRIDGE, S.M. SHAH
Cytomegalovirus (CMV) retinitis is a serious sight-threatening complication that occurs in approximately $25 \%(15-46 \%)^{1,2}$ of patients with acquired immunodeficiency syndrome (AIDS). The retinitis is characterised by necrosis, haemorrhage and eventually opacification, occurring typically with a paucity of inflammatory signs in the vitreous or in the anterior chamber. The well-known causes of decreased visual acuity in patients with AIDS and CMV retinitis include retinal detachment, ${ }^{3}$ papillitis, optic nerve atrophy ${ }^{4,5}$ and retinal necrosis with zone 1 macular involvement. Macular serous exudation in the presence of zone 1 disease has also been reported as causing reversible visual loss. ${ }^{6}$

New drug treatments such as cidofovir for CMV retinitis and protease inhibitors for HIV infection appear to be associated with a more prominent intraocular inflammatory response. Cidofovir dihydrate (1-[\{S\}-3-

hydrox\{phosphonomethoxy\}propyl]cytosine dihydrate, or HPMPC) is an acyclic nucleotide with anti-CMV activity. Both intravenous and intravitreal injection of cidofovir is associated with uveitis and ocular hypotony. ${ }^{7,8}$ The current treatment for HIV disease is highly active antiretroviral therapy (HAART). This regimen is a combination of two nucleoside analogues (dideoxyinosine, dideoxycytidine, stavudine, lamivudine) with one protease inhibitor (ritinavir, indinavir, saquanavir, nelfinavir). This therapy blocks reverse transcriptase and competitively inhibits cleavage of polyproteins of human immunodeficiency virus (HIV)-1. Consequently it prevents and decreases HIV-RNA replication and increases the $\mathrm{CD}^{+}$ count. Many recent studies ${ }^{9,10}$ have reported vitritis as a result of enhanced immune function following the introduction of protease inhibitors. As a direct consequence of inflammation, the pattern of visual morbidity in CMV retinitis in AIDS has altered immensely. The aim of this study was to investigate the causes of decreased visual acuity in CMV retinitis in patients with AIDS. 


\section{Materials and methods}

All the HIV-positive patients who attended the dedicated HIV ophthalmology clinics at two units over a 15 month period from September 1996 were reviewed. These patients had been referred from the Infection and Immunity Unit at St Bartholomew's Hospital and HIV Medicine Unit at North Middlesex Hospital when they met the following criteria: (1) visual symptoms such as blurred vision, floaters, red or painful eye, (2) any ocular signs detected on fundoscopy, (3) diagnosis of systemic $\mathrm{CMV}$, (4) $\mathrm{CD}^{+}$count of less than 100 cells/ $\mu \mathrm{l}$, (5) undiagnosed illness with opportunistic infections in HIV-positive patients (to exclude ocular disease that may help in diagnosis). Only those diagnosed as having CMV retinitis were included in this retrospective study. All patients had a detailed ophthalmic examination and in addition those with CMV retinitis underwent serial fundus photography. Serial $\mathrm{CD}^{+}$counts and viral loads were noted. Details of treatment for CMV retinitis and anti-retroviral therapy were recorded. In the two institutions involved in this study HAART was introduced in October 1996. It was prescribed in patients with low or decreasing $\mathrm{CD}^{+}$counts $(<350$ cell $/ \mu \mathrm{l})$, a high or rising viral load (10 000 copies $/ \mathrm{ml})$, new opportunistic infections or according to patient desire to start the treatment regimen. Cidofovir was available from January 1997 and the estimation of HIV viral loads, from June 1997 onwards. Decreased visual acuity was defined as best corrected visual acuity $\leqslant 6 / 12$.

\section{Results}

Of 110 patients seen in the 15 month period, 26 patients (41 eyes) were diagnosed as having CMV retinitis. There were 25 men and 1 woman. The age distribution of our patients was 32-58 years with a mean age of 38 years. The time period from the HIV-positive status to the development of CMV retinitis ranged from 1 month to 144 months with mean of 54.4 months. Twelve of 26 patients (16 eyes) with CMV retinitis had a visual acuity of $\leqslant 6 / 12$. The distribution of decreased visual acuity in

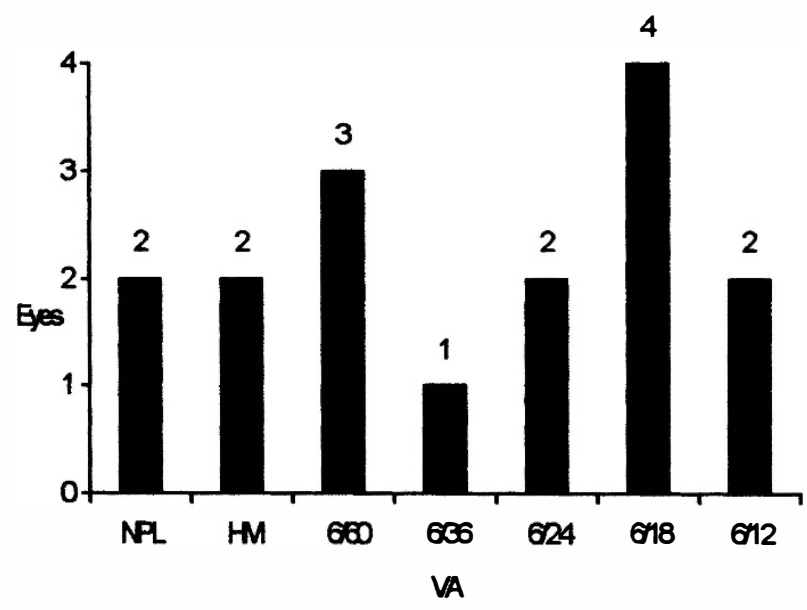

Fig. 1. Distribution of decreased visual acuity in the 16 eyes with CMV retinitis.

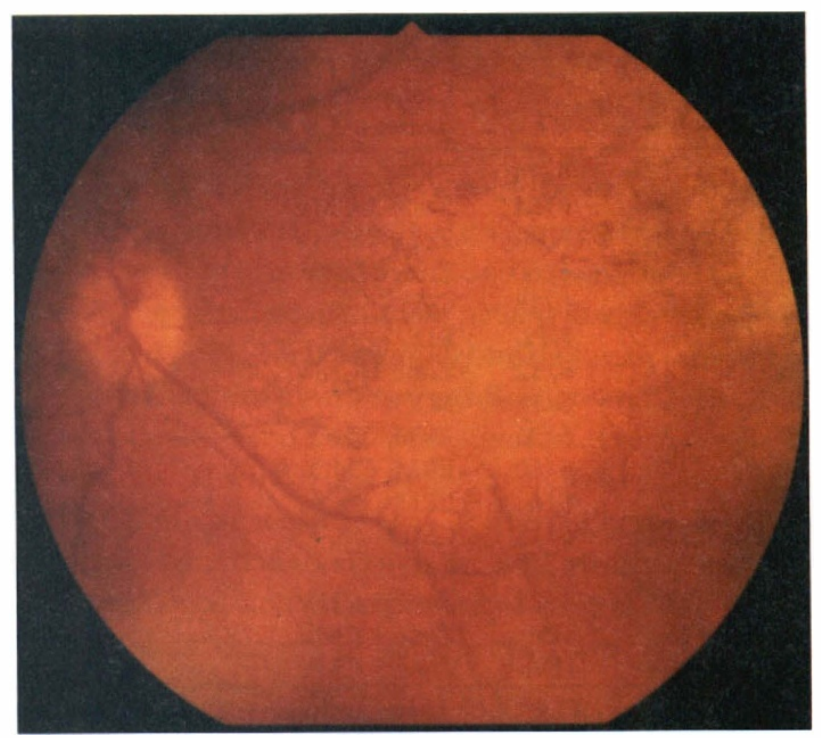

Fig. 2. Epiretinal membrane formation in $C M V$ retinitis maculopathy (patient 9).

16 eyes is shown in Fig. 1. The decreased visual acuity was due to zone $1 \mathrm{CMV}$ retinitis causing macular necrosis in 7 eyes, retinal detachment in 2 eyes and optic nerve atrophy in 1 eye. In the remaining 6 eyes ( 4 patients), decreased visual acuity was due to maculopathy, i.e. cystoid macular oedema and/or epiretinal membrane formation (Fig. 2) in the presence of inactive peripheral zone 2 or zone $3 \mathrm{CMV}$ retinitis (Table 1).

All the 4 patients in this group were on HAART and exhibited a uveitic response with cells both in the anterior chamber and in the vitreous (Table 2). Three of these 4 patients were also on concurrent cidofovir therapy and in all these the uveitis was moderate with $(2+)$ cells in the anterior chamber and vitreous. The decrease in visual acuity in these 3 patients ( 4 eyes) was progressive and finally irreversible, occurring 4-18 weeks after initiation of intravenous cidofovir. The posterior synechiae and vitreous haze and bilateral cataract in one of these patients precluded fundus photography. The intraocular pressure in these eyes was within the normal range. The remaining 1 patient on HAART therapy alone had a mild uveitis response and bilateral macular oedema (confirmed on fluorescein angiography; Fig. 3). Twenty-nine weeks after starting the treatment the $\mathrm{CD}^{+}{ }^{+}$count in 3 of 4 patients (one death in this group) increased from a mean of 27.5 (range $10-60$ ) to 112.5 (range $10-170$ ) cells $/ \mu 1$. The HIV loads decreased 3-6 months following institution of HAART therapy from a mean of 136875 (range $60000-200000$ ) to 41000 (range 42-65 170) copies/ml.

Table 1. The causes of decreased visual acuity in 12 patients (16 eyes) with $C M V$ retinitis

\begin{tabular}{ll}
\hline Zone 1 CMV & 7 eyes $(43 \%)$ \\
Retinal detachment & 2 eyes $(12 \%)$ \\
Optic nerve atrophy & 1 eye $(6 \%)$ \\
Maculopathy without zone 1 retinitis & 6 eyes $(38 \%)$ \\
\hline
\end{tabular}


Table 2. Clinical characteristics of 12 patients (16 eyes) with decreased visual acuity

\begin{tabular}{|c|c|c|c|c|c|c|c|c|c|c|}
\hline \multirow{2}{*}{$\begin{array}{l}\text { Patient } \\
\text { no. }\end{array}$} & \multirow[b]{2}{*}{ Eye no. } & \multirow[b]{2}{*}{ VA } & \multirow{2}{*}{$\begin{array}{l}\text { Cause of } \\
\text { decreased VA }\end{array}$} & \multirow[b]{2}{*}{ Uveitis } & \multirow{2}{*}{$\begin{array}{l}\text { Treatment } \\
\text { for CMV }\end{array}$} & \multirow[b]{2}{*}{ HAART } & \multicolumn{2}{|c|}{$\mathrm{CD}^{+}$} & \multicolumn{2}{|c|}{ HIV viral load (copies/ml) } \\
\hline & & & & & & & Pre & Post & Pre & Post \\
\hline 1 & 1 & $6 / 18$ & ERM & ++ & CDV & + & 60 & 100 & 200000 & 58000 \\
\hline 2 & 2 & $6 / 24$ & Zone $1 \mathrm{CMV}$ & - & Gcv, fos & - & $<10$ & $<10$ & NA & NA \\
\hline 3 & 3 & NPL & Zone $1 \mathrm{CMV}$ & - & Gcv, fos & - & $<10$ & $<10$ & NA & NA \\
\hline 3 & 4 & $6 / 60$ & Zone 1CMV & - & Gcv, fos & - & $<10$ & $<10$ & NA & NA \\
\hline 4 & 5 & $6 / 18$ & $\mathrm{OA}$ & - & Gcv, fos & - & 50 & 50 & 40000 & 58000 \\
\hline 5 & 6 & NPL & RD & - & Gcv, fos & - & $<10$ & $<10$ & NA & NA \\
\hline 6 & 7 & $6 / 12$ & Zone $1 \mathrm{CMV}$ & - & Gcv, fos & + & 15 & 140 & NA & NA \\
\hline 7 & 8 & $6 / 18$ & RD & - & Gcv, fos & + & 10 & 370 & 58000 & 40000 \\
\hline 8 & 9 & $6 / 36$ & ERM, CMO & ++ & Gcv, CDV & + & $<20$ & 170 & 150630 & 65170 \\
\hline 8 & $10^{\mathrm{a}}$ & $6 / 60$ & ERM, CMO & ++ & Gcv, CDV & + & $<20$ & 170 & 150630 & 65170 \\
\hline 9 & $11^{\mathrm{a}}$ & $6 / 60$ & ERM & ++ & Gcv, CDV & + & 10 & 10 & 900000 & deceased \\
\hline 10 & $12^{\mathrm{a}}$ & $6 / 18$ & $\mathrm{CMO}$ & + & Nil & + & 20 & 170 & 58000 & 42 \\
\hline 10 & $13^{\mathrm{a}}$ & $6 / 12$ & $\mathrm{CMO}$ & + & Nil & + & 20 & 170 & 58000 & 42 \\
\hline 11 & 14 & $6 / 24$ & Zone $1 \mathrm{CMV}$ & - & $\mathrm{Gcv}$ & - & $<10$ & $<10$ & NA & NA \\
\hline 12 & 15 & HM & Zone $1 \mathrm{CMV}$ & - & Gcv, fos & - & $<10$ & $<10$ & NA & NA \\
\hline 12 & 16 & HM & Zone $1 \mathrm{CMV}$ & - & Gcy, fos & - & $<10$ & $<10$ & NA & NA \\
\hline
\end{tabular}

VA, visual acuity; OA, optic nerve atrophy; RD, retinal detachment; CMV, cytomegalovirus; CMO, cystoid macular oedema; ERM, epiretinal membrane; CDV, cidofovir; Gcv, ganciclovir; fos, foscarnet; NA, not available as patient died before viral load assessment test was available. ${ }^{a}$ Eyes with maculopathy.

Of the 7 eyes with zone $1 \mathrm{CMV}$ retinitis involving the macula and/or optic nerve only 1 patient was on HAART therapy. He developed zone $1 \mathrm{CMV}$ retinitis

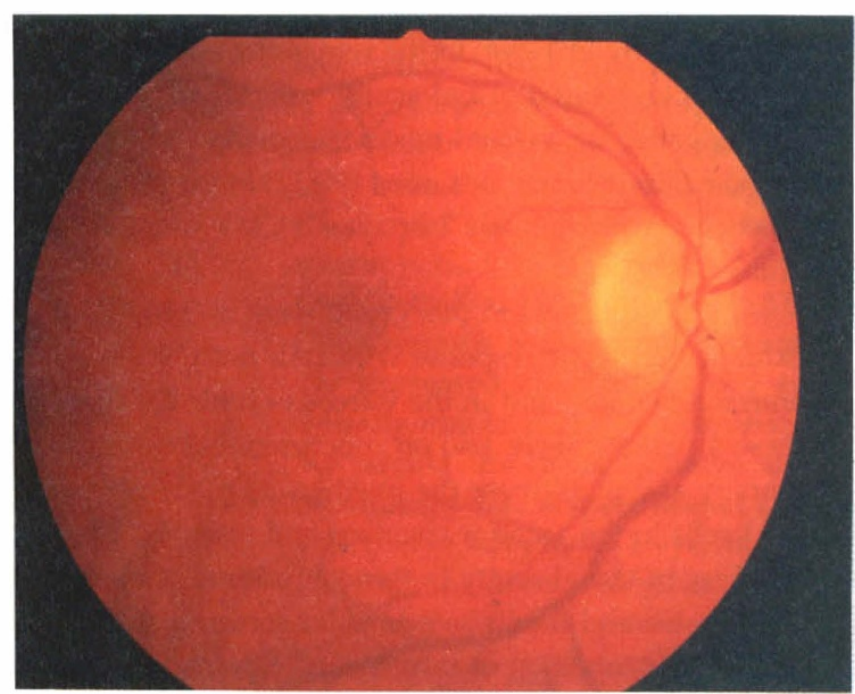

prior to being started on HAART therapy. All these patients were immunosuppressed with a $\mathrm{CD}^{+}$count $<50$ cells $/ \mu \mathrm{l}$ and none developed uveitis. One of the

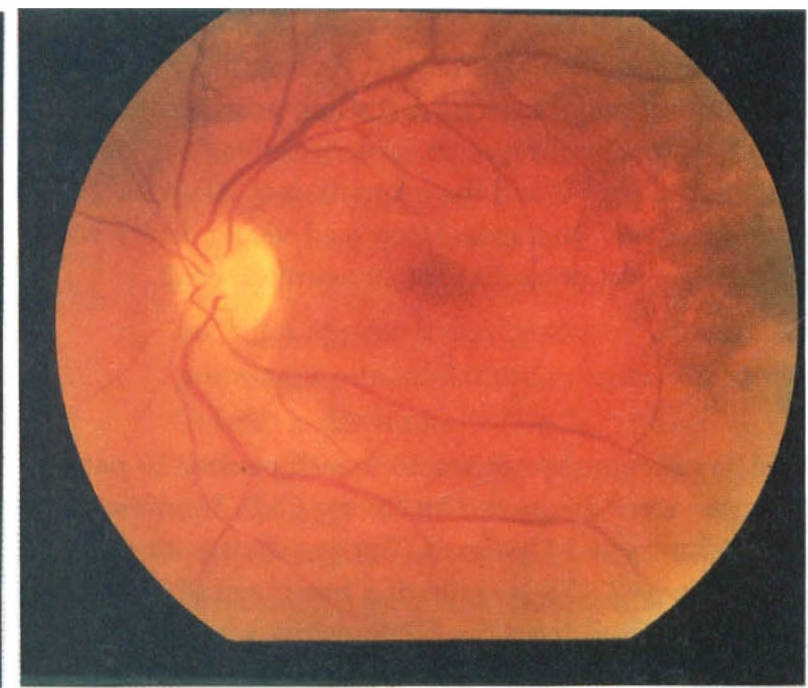

(a)
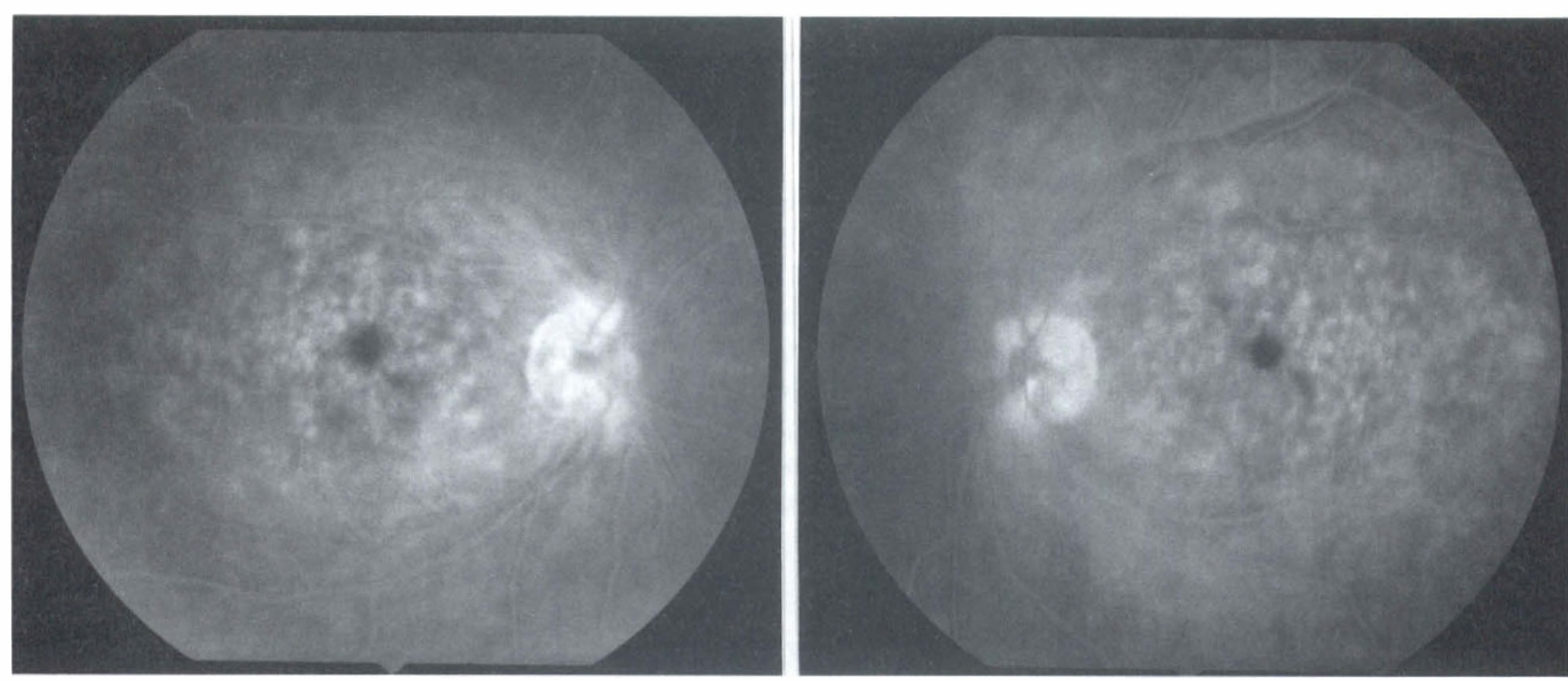

(b)

Fig. 3. (a) Fundus photographs of a patient (no. 10) with bilateral cystoid macular oedema. (b) Fluorescein angiograms of the same patient showing diffuse posterior pole leakage with cystoid macular oedema. 
patients with decreased visual acuity due to zone 2 CMV-retinitis-related retinal detachment was later started on HAART therapy and had the most impressive rise in $\mathrm{CD}^{+}{ }^{+}$counts with corresponding decrease in HIV viral loads but did not show a uveitic response (patient 7).

Of the other 14 of 26 patients with CMV retinitis and visual acuity better than $6 / 12,7$ died and none of these was on HAART therapy. Four of the 7 surviving patients are on HAART therapy and all have exhibited a lowgrade vitritis but without macular involvement.

\section{Discussion}

In this study zone $1 \mathrm{CMV}$ retinitis (7 eyes), retinal detachment ( 2 eyes) and optic nerve atrophy ( 1 eye) caused decreased visual acuity in 10 of 16 eyes $(62 \%)$. These patients were the typical immunosuppressed patients of the pre-HAART era. ${ }^{11}$ Maculopathy was the other leading cause of visual loss in 6 of 16 eyes (38\%) in the other group of patients ( 3 of 4 ) with immune recovery following HAART treatment. This alteration in the clinical characteristics of CMV retinitis in AIDS patients is related to ocular inflammation and has paralleled the changes in their immune status during the period of transition to new treatment regimens.

The potential risk factors for the development of inflammation in our patients are protease inhibitors as a part of triple therapy and intravenous cidofovir. None of these patients was on rifabutin, ${ }^{12}$ which is also known to cause anterior uveitis, during or 2 months prior to the study.

Many recent reports have commented on associated intraocular inflammation since the introduction of protease inhibitors in HIV patients. ${ }^{9,10}$ The drugs appear to result in an enhanced immune response as evidenced by increasing $\mathrm{CD}^{+}$counts and decreasing HIV viral load. This 'immune restitution' ${ }^{9}$ may, in turn, lead to an inflammatory response to $\mathrm{CMV}$, increasing the potential for development of maculopathy. Intravenous cidofovir has been documented in recent reports to cause uveitis ${ }^{13}$ and ocular hypotony, ${ }^{7}$ probably due to an effect on the ciliary body non-pigmented epithelium. While the precise mechanism of its action is unknown, these sideeffects are more marked with the higher dosage of intravitreal injections. The possibility of an interaction between cidofovir and protease inhibitors as a cause of increased inflammation has also been raised. ${ }^{7}$

The common pathway for causing non-specific macular oedema is the breakdown of the blood-retinal barriers. Elevated laser flare photometry readings in eyes with CMV retinitis also suggest that a breakdown of the blood-ocular barrier is already present in these patients. ${ }^{14}$ In the maculopathy patients this breakdown occurred due to enhanced ocular inflammation, which was clinically manifested in these eyes as anterior chamber activity, posterior synechiae and vitritis. Two of these eyes rapidly developed cataract within 3 months of onset of uveitis. Our patients were already on HAART for 8-10 weeks before the initiation of intravenous cidofovir. The onset of uveitis was seen $4-10$ weeks after initiation of cidofovir.

In 3 of the 4 maculopathy patients who were on concurrent treatment the uveitic response was severe and rapid in comparison with the one patient on HAART only. The visual acuity in this patient fluctuated between $6 / 12$ and $6 / 36$. He was initially treated with topical nonsteroidal anti-inflammatory medication and oral acetazolamide and later with steroidal orbital floor injections. The latter led to clinical improvement and stability of visual acuity at $6 / 18$. The patients with uveitis on cidofovir continued with the treatment albeit at a reduced dose, and were prescribed topical steroids with partial improvement in the anterior chamber inflammatory response. None of these patients, however, had ocular hypotony and the associated maculopathy.

The $\mathrm{CD}^{+}$counts in 3 of the 4 patients (one deceased) showed a mean increase from 27.5 to 112.5 cells / $\mu$ l and a corresponding decrease in HIV-RNA load from a mean

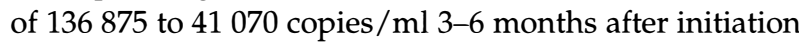
of HAART. None of these patients developed recurrence of CMV retinitis while on HAART and cidofovir during the study period. While it is difficult to separate the inflammatory effects of cidofovir and protease inhibitors because of the small numbers of patients in this study, the introduction of cidofovir appeared to be associated with the onset of uveitis. This, coupled with improved immune function, exacerbated ocular inflammation hastening the breakdown of blood-ocular barriers and hence the development of maculopathy. In this context it may be prudent to use cidofovir judiciously, especially in patients on concurrent protease inhibitors, to prevent the exaggerated inflammatory response and its subsequent effect on the macula and visual acuity.

Macular oedema in CMV retinitis in AIDS patients is uncommon as these patients are notably immunosuppressed and factors other than CMVretinitis-induced inflammation have previously been implicated as a cause of maculopathy in this group of patients. Lalonde et al. ${ }^{15}$ reported a case of zidovudinerelated cystoid macular oedema which resolved after discontinuation of medication. Palestine and Frishberg ${ }^{16}$ reported clinical macular oedema in patients with inactive CMV retinitis in AIDS which was attributed to microvascular retinopathy. Weinberg and Moorthy ${ }^{17}$ reported a case of unilateral cystoid macular oedema in CMV retinitis in AIDS. The patient was on rifampicin, had zone $1 \mathrm{CMV}$ retinitis, was not on protease inhibitors and his $\mathrm{CD}^{+}$count was only 3 cells $/ \mu$ l.

Maguire et al. ${ }^{18}$ reported cystoid macular oedema and vitritis in 4 eyes with CMV retinitis in patients without AIDS and suggested that the lack of inflammatory response in grossly immunosuppressed AIDS patients seemed to protect them from acquiring cystoid macular oedema. Silverstein and associates ${ }^{19}$ also reported cystoid macular oedema in patients with inactive CMV retinitis in AIDS. However, none of these patients was on HAART therapy. Our findings of maculopathy appear to be similar to those reported by Karavellas et al., ${ }^{20}$ who 
label the associated vitritis as an 'immune recovery vitritis'; more recently it has been described by Cassoux et $a .^{21}$ as due to immune restoration following HAART. None of these patients was on concurrent cidofovir.

In summary, our study highlights that CMV-related maculopathy, as a response to the effects of therapy for CMV and HIV rather than a direct consequence of CMV retinitis, is a significant cause of visual morbidity. With new drug regimens for both HIV and CMV resulting in an increasing survival and a decreasing incidence of new or active CMV retinitis, ${ }^{22,23}$ maculopathy as a cause of decreased visual acuity is likely to pose important management challenges.

\section{References}

1. Holland GN, Pepose JS, Pettit TH, et al. Acquired immune deficiency syndrome: ocular manifestations. Ophthalmology 1983;90:859-73.

2. Jabs DA, Enger C, Bartlett JG. Cytomegalovirus retinitis and immune deficiency syndrome. Arch Ophthalmol 1989;107:75-80.

3. Freeman WR, Henderly DE, Wan WL, et al. Prevalence, pathophysiology, and treatment of rhegmatogenous retinal detachment in treated cytomegalovirus retinitis. Am J Ophthalmol 1987;103:527-36.

4. Gross JG, Sadun AA, Wiley CA, Freeman WR. Severe visual loss related to isolated peripapillary retinal and optic nerve head cytomegalovirus infection. Am J Ophthalmol 1989;108:691-8.

5. Grossniklaus HE, Frank KE, Tomask RL Cytomegalovirus retinitis and optic neuritis in acquired immune deficiency syndrome. Ophthalmology 1987;94:1601-4.

6. Gangan PA, Besen G, Munguia D, Freeman WR. Macular serous exudation in patients with acquired immunodeficiency syndrome and cytomegalovirus retinitis. Am J Ophthalmol 1994;118:212-9.

7. Davis JL, Taskintuna I, Freeman WR, et al. Iritis and hypotony after treatment with intravenous cidofovir for cytomegalovirus retinitis. Arch Ophthalmol 1997;115:733-7.

8. Jabs DA. Cidofovir [review]. Arch Ophthalmol 1997;115:785-6.

9. Nussenblatt RB, Lane HC. Human immunodeficiency virus disease: changing patterns of intraocular inflammation. Am J Ophthalmol 1998;125:374-82.
10. Zegans ME, Walton RC, Holland GN, et al. Transient vitreous inflammatory reactions associated with combination antiretroviral therapy in patients with AIDS and cytomegalovirus retinitis. Am J Ophthalmol 1998;125:292-300.

11. Roarty JD, Fisher EJ, Nussbaum JJ. Long term visual morbidity of cytomegalovirus retinitis in patients with acquired immunodeficiency syndrome. Ophthalmology 1993;100:1685-8.

12. Tseng AL, Walmsley SL. Rifabutine-associated iritis. Ann Pharmacother 1995;29:1149-55.

13. Bainbridge JWB, Raina J, Shah SM. Anterior uveitis associated with CMV retinitis in AIDS: a review. Invest Ophthalmol Vis Sci 1998;39:S134.

14. Magone MT, Nussenblatt RB, Whitcup SM. Elevation of laser photometry in patients with cytomegalovirus retinitis and AIDS. Am J Ophthalmol 1997;124:190-8.

15. Lalonde RG, Deschenes JG, Seamone C. Zidovudine-induced macular edema. Ann Intern Med 1991;114:297-8.

16. Palestine AG, Frishberg B. Macular edema in acquired immunodeficiency syndrome related microvasculopathy. Am J Ophthalmol 1991;111:770-1.

17. Weinberg DV, Moorthy RS. Cystoid macular edema due to cytomegalovirus retinitis in patients with acquired immunodeficiency syndrome. Retina 1996;16:343-4.

18. Maguire AM, Nichols CW, Crook GW. Visual loss in cytomegalovirus retinitis caused by cystoid macular edema in patients without the acquired immunodeficiency syndrome. Ophthalmology 196;103:601-5.

19. Silverstein BE, Smith JH, Sykes SO, et al. Cystoid macular edema associated with cytomegalovirus retinitis in patients with acquired immunodeficiency syndrome. Am J Ophthalmol 1998;25:411-5.

20. Karavellas MP, Lowder CY, Macdonald JC, et al. Immune recovery vitritis associated with inactive cytomegalovirus retinitis. Arch Ophthalmol 1998;116:169-75.

21. Cassoux N, Lumbroso L, Bodaghi B, et al. Cystoid macular oedema and cytomegalovirus retinitis in patients with HIV disease treated with highly active antiretroviral therapy. Br J Ophthalmol 1999;83:47-9.

22. Jabs DA, Bartlett JG. AIDS and ophthalmology: a period of transition [editorial]. Am J Ophthalmol 1997;124:227-33.

23. Lee V, Subak-Sharpe I, Shah S, et al. The changing trends in cytomegalovirus retinitis with triple therapy. Eye 1999;13:59-64. 